\title{
Pupillography of automated swinging flashlight test in amblyopia
}

\author{
Atsushi Miki' \\ Atsuhiko lijima ${ }^{2}$ \\ Mineo Takagi' \\ Kiyoshi Yaoeda' \\ Tomoaki Usui' \\ Shigeru Hasegawa' \\ Haruki Abe' \\ Takehiko Bando ${ }^{2}$ \\ 'Department of Ophthalmology; \\ ${ }^{2}$ Department of Physiology, Niigata \\ University Graduate School of \\ Medical and Dental Sciences, Niigata, \\ Japan
}

\begin{abstract}
Relative afferent pupillary defects (RAPDs) in amblyopia have been reported, and it is widely accepted that amblyopes can have an RAPD. We investigated whether or not this could be confirmed by the use of binocular pupillography. We examined twelve patients (6 males and 6 females, aged 7-57 years) with unilateral amblyopia associated with anisometropia and/or strabismus, using binocular infrared video pupillography (Newopto, Kawasaki, Japan). Eight normal subjects were also tested in the same manner. Two patients' data had to be excluded because of poor recording quality. Only one patient with moderate anisometropic amblyopia was found to have reduced contraction amplitude in the amblyopic eye, and one patient with a borderline pupillary defect. The other amblyopes, some of whom showed even denser amblyopia, did not have a pupillary defect. This study has confirmed that only a small proportion of amblyopes have a reduced pupillary contraction amplitude in the affected eye, as established by pupillographic recordings, and even these amblyopes are not necessarily associated with dense amblyopia.
\end{abstract}

Keywords: relative afferent pupillary defect, amblyopia, pupillography

\section{Introduction}

It is widely accepted that patients with unilateral amblyopia can have a relative afferent pupillary defect (RAPD). There have been many reports demonstrating a reduction in the pupil response for amblyopic eyes (Brenner 1969; Greenwald and Folk 1983; Portnoy et al 1983; Firth 1990; Barbur et al 1994; Donahue 1997). Since the pupillomotor efferents were found to be normal (Kase et al 1984; Barbur et al 1994), the pupillary deficits must lie in the afferent pupillary pathway. However, the proportion of the amblyopes which showed RAPD varies considerably across clinical studies (Greenwald and Folk 1983; Portnoy et al 1983). Moreover, no significant decrease in the contraction amplitude in amblyopic eyes was reported (Kase et al 1984). No correlation between the depth of amblyopia (visual acuity) and the presence of RAPD or the amount of RAPD has been reported (Greenwald and Folk 1983; Portnoy et al 1983). Also, the reported RAPD in amblyopia is usually fairly small ( $\leq 0.6 \log$ unit) (Portnoy et al 1983), making it difficult to determine whether it is pathological or within normal variation, especially because normal subjects have been shown to have small RAPDs (Kawasaki et al 1995, 1996). These observations have caused some researchers to cast doubt on the presence of RAPD in unilateral amblyopia.

Although pupillary tests are basically objective in that they do not require answers from patients, the determination of the endpoint in the swinging- flashlight test with neutral-density filters can be quite subjective. Thus, an objective measurement of pupillary response is desirable in order to establish whether light reflex is affected in amblyopia. Therefore, we recorded binocular pupillography in patients with unilateral amblyopia during an automated swinging-flashlight test in order to investigate whether or not pupillary defects could be demonstrated objectively. 
Previous studies using pupillography have revealed that RAPD up to $0.3 \log$ unit can be observed in normal subjects and, therefore, should be considered benign (Kawasaki et al 1996). The difference in contraction amplitude corresponding to $0.3 \log$ unit was found to be approximately $0.25 \mathrm{~mm}$ in the previous studies (Kawasaki et al 1995, 1996; Volpe et al 2000). Therefore, because we did not measure the RAPD with neutral density filters in this study, we set the cut-off value at $0.25 \mathrm{~mm}$ of the difference in contraction amplitude. We also investigated whether or not this threshold worked for normal subjects.

\section{Methods}

Twelve patients with unilateral strabismic and/or anisometropic amblyopia (6 men and 6 women, aged 7-57 years) were examined. The visual acuity of the amblyopic eye, assessed using the optotype test, ranged from 20/200 to 20/30. The visual acuity of the fellow eye was 20/20 or better in all the patients. Eight age-matched normal subjects with a visual acuity of 20/20 or better in each eye ( 2 men and 8 women, aged $10-53$ years) were also tested in the same fashion and served as controls. Written informed consent was obtained from each patient. This study was approved by the institutional review board of Niigata University Hospital. None of the subjects were paid for their participation in this study. In three patients, the examination was repeated on a different day to confirm the results.

Pupillary responses were measuredwith a binocular infrared video pupillographic device (Vision module, Newopto, Kawasaki, Japan) in a darkened room. Each patient was placed in the darkened room for approximately six minutes before the measurement so that they could adapt to the darkness. The horizontal pupillary diameter was recorded 30 times per second. Light stimuli with white light-emitting diodes (LEDs) were presented to the right and left eyes alternatively. The LEDs' position was adjusted for each eye tested. Care was taken to position the LEDs by visual inspection so as to shine the light along the visual axis for each eye. The subjects were instructed to look at distance to avoid accommodative miosis. The timing of visual stimulation by the LEDs for each eye was controlled by the same computer that recorded the pupillary responses (Miki et al 2006, 2008a). Two stimulus-durations were used for the automated swinging-flashlight test: 1) 0.2 seconds of light on and 0.8 seconds of light off, and 2) 0.3 seconds of light on and 0.95 seconds of light off. The patients were instructed to try to refrain from blinking during the test.
The contraction amplitude was defined as the difference between maximum and minimum diameters. In order to correct for the effect of contraction anisocoria, the average of the constriction amplitudes of both eyes (ie, the direct and indirect pupillary light reflexes) was calculated to represent the pupillary response to light stimulation to each eye. The average values for the four right-eye stimulations were compared with those of the four left-eye stimulations. The presence of fixation instability was closely investigated in order to exclude unreliable data, especially those due to poor fixation by the amblyopic eyes.

\section{Results}

Data from two patients (a 9-year-old girl and an 18-year-old man) had to be excluded because of poor recording quality, possibly due to poor fixation or narrow palpebral fissure. Measurements of all the other ten patients and normal subjects were successfully completed.

As a group, the difference in contraction amplitude in amblyopic subjects was indistinguishable from that of normal subjects ( $p=0.4492$, Mann-Whitney test; Figure 1). There was no significant correlation between the visual acuity of the amblyopic eye and the difference in contraction amplitude ( $\mathrm{p}=0.3965$, Spearman's rank correlation; Figure 2). The difference in contraction amplitude was smaller than $0.25 \mathrm{~mm}$ in all of the normal subjects. Some patients showed a small "pupillary defect" in the non-amblyopic eye; this was considered to be insignificant and benign.

Only one patient with anisometropic amblyopia showed a reduced contraction amplitude in the affected eye; moreover, it was reproduced on a different day (Figure 3). She did not have manifest strabismus, and the density of her amblyopia in the left eye was mild. The reduced contraction amplitude observed in the patient could not be attributed to the effect of patching because the patient had not been treated with patching. In addition, the patient's fixation was stable during the measurement. Another patient with anisometropic and strabismic amblyopia was considered to have a "borderline" abnormality (Figure 4). Although some of the patients had dense amblyopia, none of the other amblyopes showed a pupillary defect (Figure 5).

\section{Discussion}

In this study, we used binocular infrared video pupillography to record light reflex in amblyopia. Contrary to some of the previous reports (Brenner et al 1969; Portnoy et al 1983), pupillary defects were not consistently observed in the amblyopic eyes. In addition, there does not seem to 


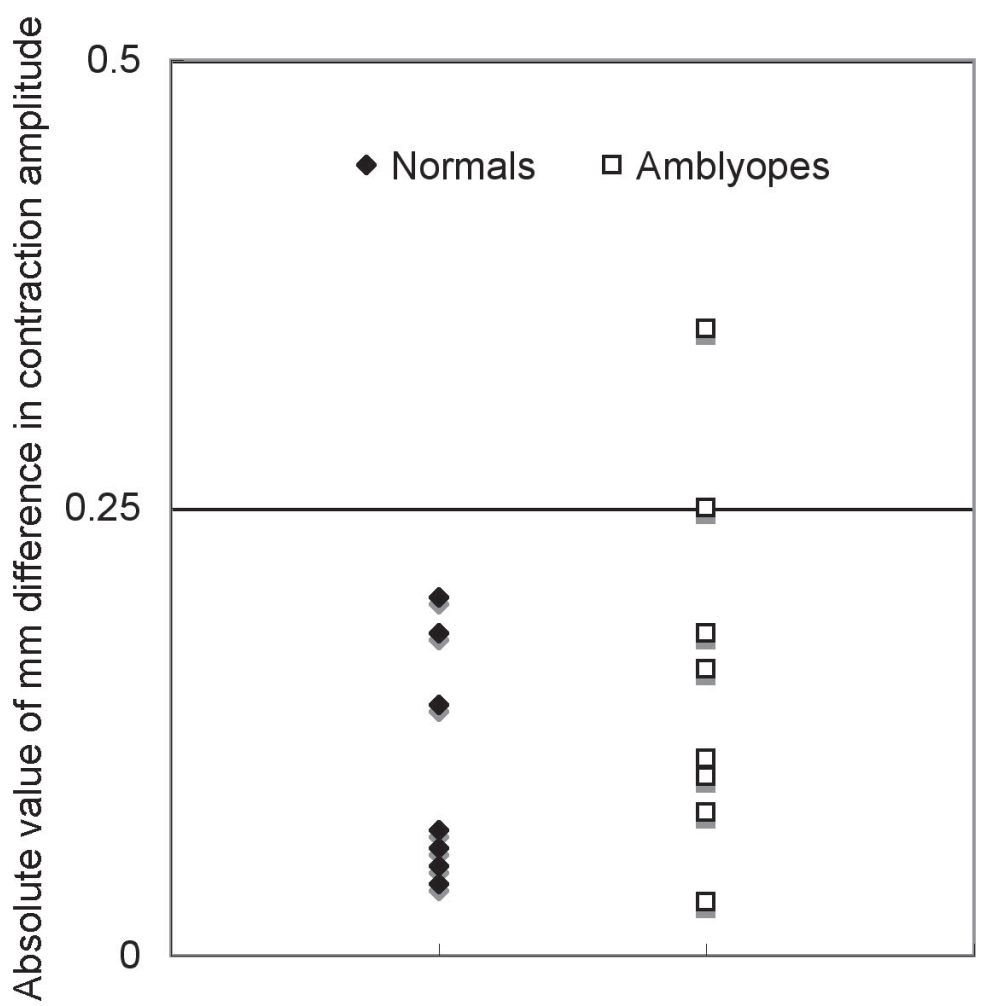

Figure I Absolute value of the intereye differences in contraction amplitude in normal subjects and in patients with unilateral amblyopia.

be a correlation between the visual acuity of amblyopic eyes and the difference in contraction amplitude of both eyes.

To learn whether or not amblyopes have RAPDs is important because it may elucidate the neural basis of the amblyopia. It is generally believed that the visual cortex is responsible for the deficits in amblyopia (Hess 2001; Barrett et al 2004), but there may also be dysfunction and/or malformation outside the visual cortex (Miki et al 2003; Lempert 2004). There is much evidence that the visual cortex in human amblyopia is anomalous (Goodyear et al 2000; Barnes et al 2001; Choi et al 2001; Algaze et al 2002; Liu et al 2004; Bonhomme et al 2006; Conner et al 2007; Miki et al 2008b), but the number of reports showing anomaly in the lateral geniculate nucleus or retina is limited. Because the primary visual cortex is the first area in the visual system where signals from both eyes are integrated, the amblyopic deficits are most likely to originate from the visual cortex rather than from the eye, especially in amblyopia caused by abnormal binocular interaction during visual development. However, there may be involvement of lateral genicualte nucleus and retina secondary to the abnormality in the visual cortex.

Our results demonstrate that pupillary defects in amblyopia are seen only in some patients and are mild even if present. The frequency of the pupillary defects in amblyopia in this report seems to be consistent with the observation by Greenwald and Folk (1983) that revealed mild RAPDs in only 4 out of 45 amblyopes. Similarly, Barbur and colleagues (1994) reported that a small number of amblyopic eyes showed smaller contraction amplitudes than the fellow eyes, but the pupillary light reflex in amblyopes was generally normal as a group. In contrast, there have been at least two previous reports that the vast majority of amblyopes showed RAPDs (Brenner et al 1969; Portnoy et al 1983). Portnoy and colleagues (1983) also reported that one patient had a fairly large RAPD of $0.9 \log$ unit, almost 4 standard deviations above the mean.

As compared with clinical swinging flashlight test, the duration of the light stimulation in this study was rather short and was chosen after some preliminary experiments. We must be aware that there is a trade-off between the length and the number of each stimulus. Unlike the clinical test, where pupillary escape can be detected in a few swings, this test requires a certain number of light alternations to obtain reliable results (Kasawaki et al 1995). Therefore, although these parameters are certainly different from the clinical one, we believe that the duration and interval of the stimulation that we used here are appropriate. Similar stimulus conditions have been used in a previous pupillographic study (Volpe et al 2000). 


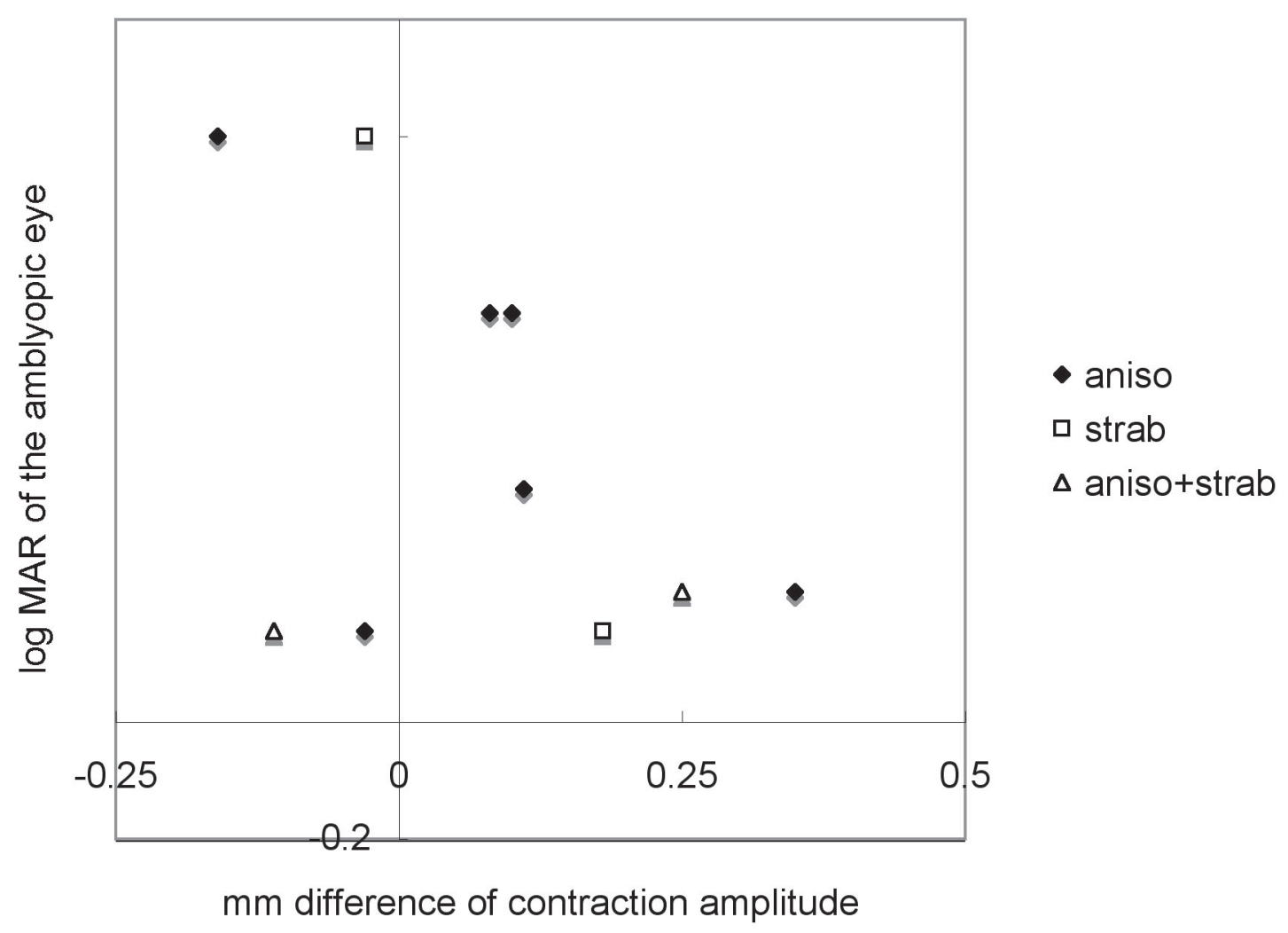

Figure 2 Visual acuity of the amblyopic eye and the difference in contraction amplitude (stimulation of non-amblyopic eye minus stimulation of amblyopic eye). Abbreviations: aniso, anisometropic amblyopia; strab, strabismic amblyopia; aniso+strab, anisometropic and strabismic amblyopia.

It is rather surprising to us that even dense amblyopes showed no pupillary defects. There have been previous reports on the discrepancy between pupillary defects including latency differences and visual acuity loss or contrast sensitivity deficit
(Greenwald and Folk 1983; Portnoy et al 1983; Kase et al 1984; Barbur et al 1994), although the contrary has also been reported (Brenner et al 1969; Firth 1990). One possible explanation of this discrepancy is that some amblyopes have

Anisometropic amblyopia OS

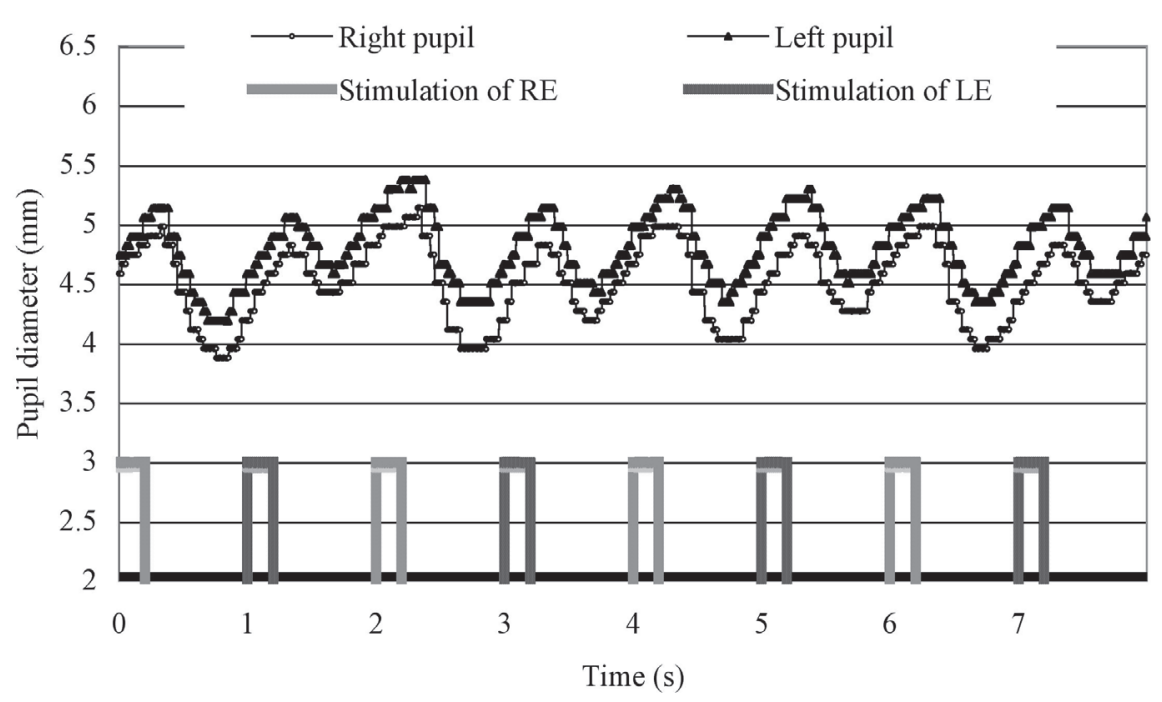

Figure 3 Pupillographic tracings of an 8-year-old girl with anisometropic amblyopia OS. The visual acuity OS was 20/30. The timing of light stimulation is shown below the pupillographic tracings. The right-eye stimulation resulted in a greater contraction than did the left-eye stimulation. 


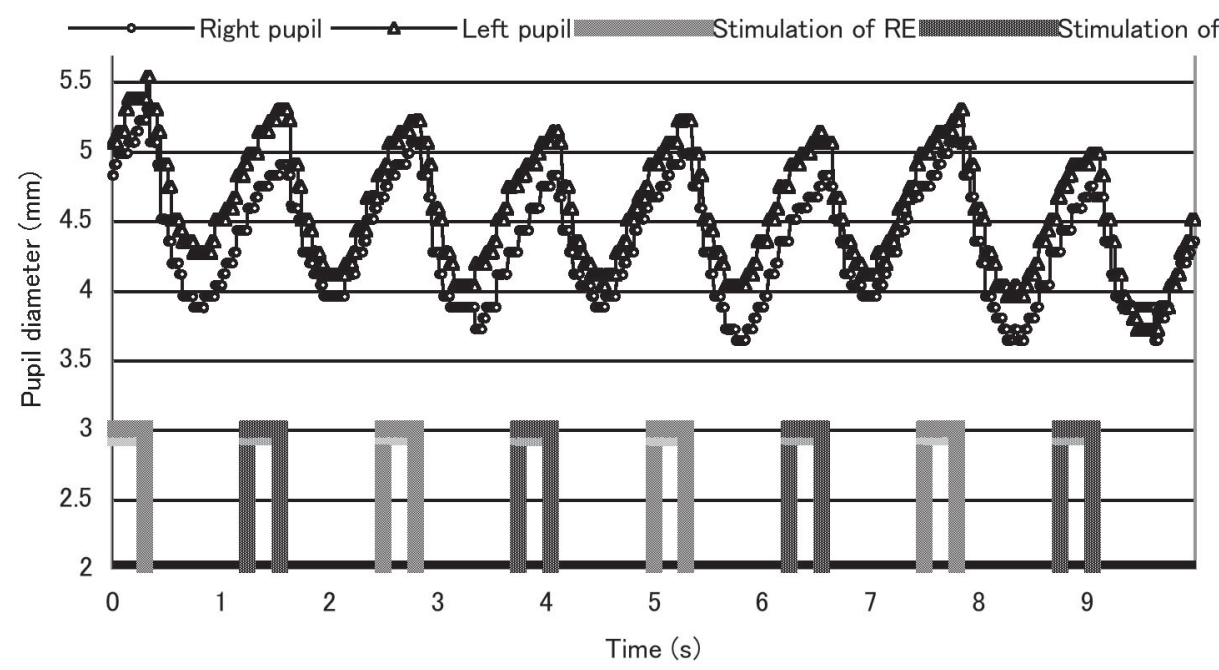

Figure 4 Pupillographic tracings of an II-year-old boy with anisometropic and strabismic amblyopia OS.The visual acuity was 20/30 in the left eye. The contraction amplitude was slightly larger when the right eye was stimulated.

abnormalities in the pupillary reflex pathway, while others do not. Suppression of the amblyopic eyes may have a role in the occurrence of RAPD (Brenner et al 1969). Although we did not investigate the effect of amblyopia treatment on the pupillary defects in amblyopia, a previous report has suggested a relationship between the period of occlusion and the presence of RAPD (Firth 1990).

The most important clinical implication of this study is that only mild abnormalities of the pupil occur on the basis of amblyopia. Therefore, when a marked RAPD is observed, diseases of the optic nerve or the retina should first be suspected.
There are limitations of our study. To determine the amount of RAPD (in terms of log unit), a series of responses to graded stimuli must be recorded (Kasawaki et al 1995, 1996). Since we measured the difference in contraction amplitude, we cannot unambiguously prove that there was no RAPD in whom we did not find such a difference. The subtle RAPD which has been reported in amblyopia may have been missed in this study. Thus, our conclusion may apply only to the contraction amplitude difference in amblyopia when tested with the stimulation condition that we used. Also, as we tested only a limited number of patients, this study may not have enough statistical power to detect a small trend.

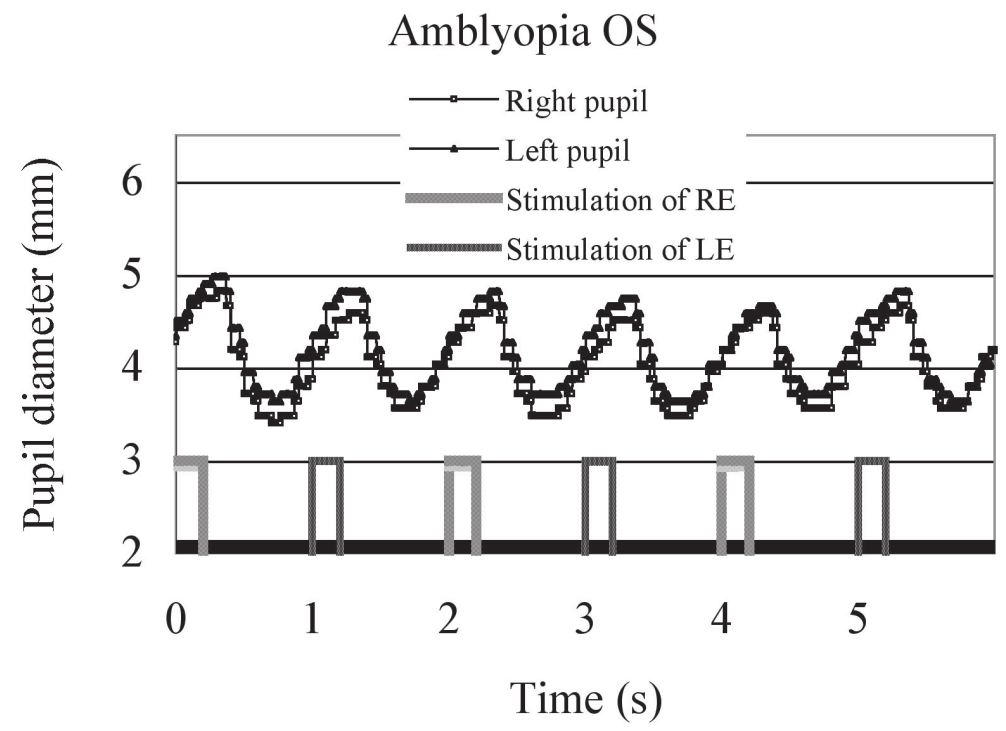

Figure 5 Pupillographic tracings of a 10-year-old girl with anisometropic and strabismic amblyopia OS.The visual acuity was $20 / 100$ in the left eye.The contraction amplitudes were similar regardless of the eye stimulated. 
The origin of RAPD in amblyopia remains unclear. Given the functional and anatomical abnormality in the visual cortex reported in many previous articles on amblyopia, supranuclear ("top-down") influences may account for the defects. This may resemble the pupillary defects observed in cortical visual disturbance (Yoshitomi et al 1999). Nonetheless, the possibility cannot be completely ruled out that the afferent pupillary pathway from the retina to the midbrain in some amblyopes is affected, possibly secondarily to anomalies in the visual cortex.

\section{Disclosure}

Presented at the Association for Research in Vision and Ophthalmology Annual Meeting, Fort Lauderdale, Florida, USA., May 9, 2007. Dr. Miki was supported by Grant for Promotion of Niigata University Research Projects. The authors report no conflicts of interest in this work.

\section{References}

Algaze A, Roberts C, Leguire L, et al. 2002. Functional magnetic resonance imaging as a tool for investigating amblyopia in the human visual cortex: a pilot study. J AAPOS, 6:300-8.

Barbur JL, Hess RF, Pinney HD. 1994. Pupillary function in human amblyopia. Ophthalmic Physiol Opt, 14:139-49.

Barnes GR, Hess RF, Dumoulin SO, et al. 2001. The cortical deficit in humans with strabismic amblyopia. J Physiol, 533:281-97.

Barrett BT, Bradley A, McGraw PV. 2004. Understanding the neural basis of amblyopia. Neuroscientist, 10:106-17.

Bonhomme GR, Liu GT, Miki A, et al. 2006. Decreased cortical activation in response to a motion stimulus in anisometropic amblyopic eyes using functional magnetic resonance imaging. J AAPOS, 10:540-6.

Brenner RL, Charles ST, Flynn JT. 1969. Pupillary responses in rivalry and amblyopia. Arch Ophthalmol, 82:23-9.

Choi MY, Lee K-M, Hwang J-M, et al. 2001. Comparison between anisometropic and strabismic amblyopia using functional magnetic resonance imaging. Br J Ophthalmol, 85:1052-6.

Conner IP, Odom JV, Schwartz TL, et al. 2007. Monocular activation of $\mathrm{V} 1$ and $\mathrm{V} 2$ in amblyopic adults measured with functional magnetic resonance imaging. $J A A P O S, 11: 341-50$.
Donahue SP, Moore P, Kardon RH. 1997. Automated pupil perimetry in amblyopia: generalized depression in the involved eye. Ophthalmology, 104:2161-7.

Firth AY. 1990. Pupillary responses in amblyopia. Br J Ophthalmol, 74:676-80.

Goodyear BG, Nicolle DA, Humphrey GK, et al. 2000. BOLD fMRI response of early visual areas to perceived contrast in human amblyopia. J Neurophysiol, 84:1907-13.

Greenwald MJ, Folk ER. 1983. Afferent pupillary defects in amblyopia. J Pediatr Ophthalmol Strabismus, 20:63-7.

Hess RF. 2001. Amblyopia: site unseen. Clin Exp Optom, 84:321-36.

Kase M, Nagata R, Yoshida A, et al. 1984. Pupillary light reflex in amblyopia. Invest Ophthalmol Vis Sci, 25:467-71.

Kawasaki A, Moore P, Kardon RH. 1996. Long-term fluctuation of relative afferent pupillary defect in subjects with normal visual function. Am J Ophthalmol, 122:875-82.

Kawasaki A, Moore P, Kardon RH. 1995. Variability of the relative afferent pupillary defect. Am J Ophthalmol, 120:622-33.

Lempert P. 2004. The axial length/disc area ratio in anisometropic hyperopic amblyopia: a hypothesis for decreased unilateral vision associated with hyperopic anisometropia. Ophthalmology, 111:304-8.

Liu GT, Miki A, Francis E, et al. 2004. Eye dominance in visual cortex in amblyopia using functional magnetic resonance imaging. J AAPOS, 8:184-6.

Miki A, Iijima A, Takagi M, et al. 2008a. Pupillography of relative afferent pupillary defects in amblyopia associated with peripapillary myelinated nerve fibers and myopia. J Pediatr Ophthalmol Strabismus, 45:309-12.

Miki A, Iijima A, Takagi M, et al. 2006. Pupillography of relative afferent pupillary defect contralateral to monocular mature cataract. Can J Ophthalmol, 41:469-71.

Miki A, Liu GT, Goldsmith ZG, et al. 2003. Decreased activation of the lateral geniculate nucleus in a patient with anisometropic amblyopia demonstrated by functional magnetic resonance imaging. Ophthalmologica, 217:365-9.

Miki A, Siegfried JB, Liu C-SJ, et al. 2008b. Magno- and parvocellular visual cortex activation in anisometropic amblyopia, as studied with functional magnetic resonance imaging. Neuro-ophthalmology, In press.

Portnoy JZ, Thompson HS, Lennarson L, et al. 1983. Pupillary defects in amblyopia. Am J Ophthalmol, 96:609-14.

Volpe NJ, Plotkin ES, Maguire MG, et al. 2000. Portable pupillography of the swinging flashlight test to detect afferent pupillary defects. Ophthalmology, 107:1913-21.

Yoshitomi T, Matsui T, Tanakadate A, et al. 1999. Comparison of threshold visual perimetry and objective pupil perimetry in clinical patients. J Neuroophthalmol, 19:89-9. 